OPEN ACCESS

Edited by:

Joelle Aubin,

Université de Toulouse, France

Reviewed by:

Kunn Hadinoto,

Nanyang Technological University,

Singapore

Xunli Zhang,

University of Southampton, United Kingdom

*Correspondence:

José Carlos B. Lopes lopes@fe.up.pt

Specialty section:

This article was submitted to Microfluidic Engineering and Process Intensification,

a section of the journal

Frontiers in Chemical Engineering

Received: 06 September 2021

Accepted: 12 October 2021

Published: 12 November 2021

Citation:

Matos J, Santos RJ, Dias MM and Lopes JCB (2021) Mixing in the

NETmix Reactor.

Front. Chem. Eng. 3:771476. doi: 10.3389/fceng.2021.771476

\section{Mixing in the NETmix Reactor}

\author{
Joana Matos, Ricardo J. Santos, Madalena M. Dias and José Carlos B. Lopes* \\ LSRE-LCM - Laboratory of Separation and Reaction Engineering-Laboratory of Catalysis and Materials, Department of \\ Chemical Engineering, Faculty of Engineering, University of Porto, Porto, Portugal
}

NETmix is a static mixing reactor composed of a network of mixing chambers interconnected by channels. The repetitive mixing pattern inside the reactor enables the use of reduced geometries to represent the NETmix network, such as the ExtendedNUB model, used in this work. Mixing in NETmix is based on the impingement of jets, issuing from channels. Inside the chambers, the jets are engulfed by dynamic vortices which can be quantified using Lagrangian techniques. Batch Lagrangian Mixing Simulation (BLMS) is based on successive injections of particles to measure the fraction of the fluids at the outlet of the mixing chambers. The distribution of the outlet fraction of particles indicates that it is possible to have nearly perfect mixing inside the NETmix chambers, depending on the dimensions of the channels and chambers. The NETmix design is here optimized in relation to the chamber diameter to channel width ratio, $D / d$. Results from BLMS show that best performance in NETmix occurs for $6.65 \leq D / d \leq 6.85$.

Keywords: NETmix Reactor, Batch Lagrangian Mixing Simulation, CFD, Mixing, Design Optimization

\section{INTRODUCTION}

NETmix is a static mixer patented in 2005 (Lopes et al., 2005). The design of the reactor is based on a network of mixing chambers interconnected by transport channels (Lopes et al., 2005). The mixing chambers are elements with a circular footprint which can be cylinders or spheres, the channels that connect the chambers are cylindrical or prismatic. The centre of these elements lays in a 2D plane.

The fluids enter the NETmix reactor through separated inlets placed at the bottom row of the network and leave through a set of outlets at the top row. Figure 1 shows the NETmix network and flow patterns from a tracer CFD simulation for a chaotic flow regime (Fonte et al., 2013). The NETmix reactor enables the control of macro and micro-mixing which can be manipulated to improve the performance of the reactor (Laranjeira et al., 2009). Moreover, the periodic nature of the NETmix design enables a direct laboratory to industrial scale by numbering-up the network (Schenk et al., 2004; Saber et al., 2010).

The first NETmix device was designed in 2005 based on a network of spherical chambers connected by cylindrical channels (Laranjeira 2005). In 2008, an industrial prototype was built at Fluidinova S.A. (fluidinova.com), for the continuous large-scale production of synthetic nano-hydroxyapatite (Lopes et al., 2006; Lopes et al., 2007; Silva et al., 2008; Gomes et al., 2009). CFD studies have shown that the mixing mechanisms in NETmix are well described from 2D simulations (Gomes 2011). In this way, other NETmix units were developed, using cylindrical chambers and prismatic channels (Gomes 2011). Nowadays the NETmix network is etched in plates, making it particularly suited for coupling other

Abbreviations: BLMS, Batch Lagrangian Mixing Simulation; CDF, Computational Fluids Dynamics; LMS, Lagrangian Mixing Simulation; NUB, NETmix Unit Block Re Reynolds number. 


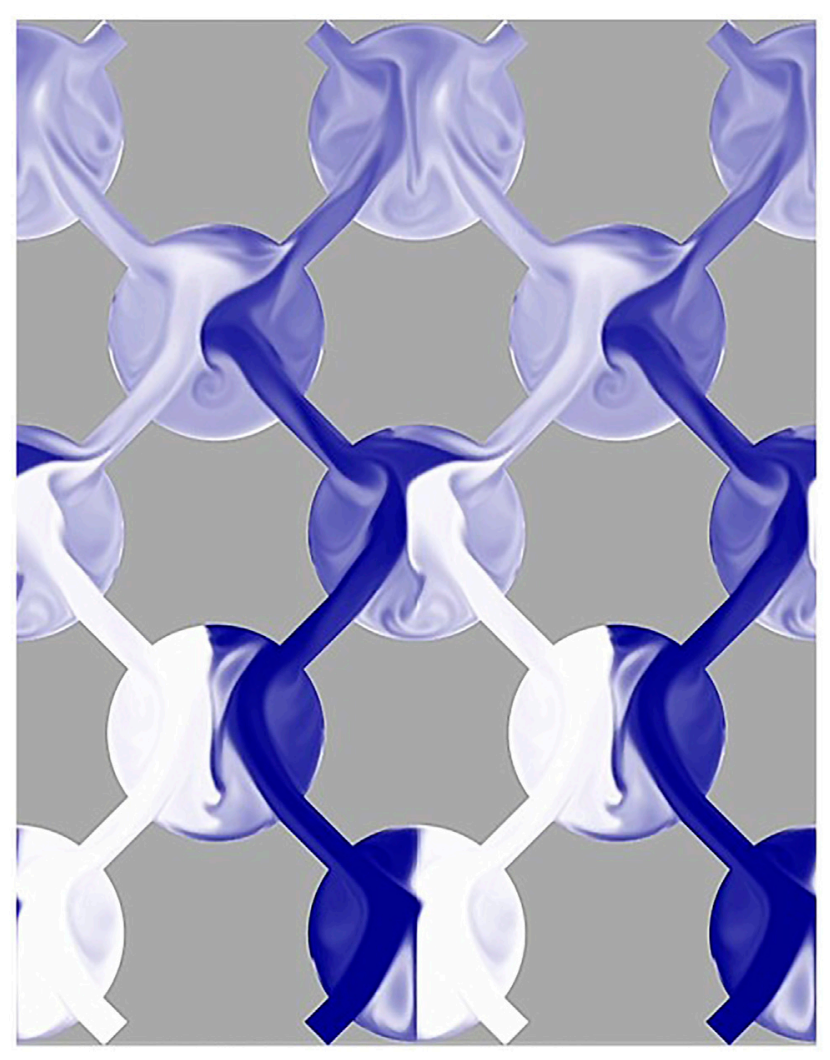

FIGURE 1 | Passive tracer concentration map: NETmix network and flow pattern (Fonte et al., 2013).

devices such as heating and radiation plates. The diameter of the chambers and channels was also varied between different NETmix devices which enables to switch from a micro to a meso device. In this way it is possible to have better control of heat and mass transfer (Costa, 2017) which has been widening the applications of this technology. Since 2016, a photocatalytic NETmix was designed (Lima et al., 2016) which is used to perform photocatalysis studies such as kinetic modelling (Marinho et al., 2017; Santos et al., 2019; Santos et al., 2021) and ozonation (Filho et al., 2019). In 2017, a NETmix device coupled to heat exchangers was developed for the continuous production of $\mathrm{CO}_{2}$ hydrates (Costa et al., 2017; Lopes et al., 2018; Lopes et al., 2019). The NETmix reactor has also been used for microencapsulation (Moreira et al., 2020) and Pickering emulsions (Ribeiro et al., 2021).

Figure 1 shows the mixing patterns inside the NETmix reactor which are repetitive along each row of mixing chambers. In this way, it is possible to study how mixing occurs inside the NETmix chambers, i.e., micromixing, through the simulation of a reduced domain of the network (Fonte et al., 2013; Costa et al., 2017), replacing the full network by periodic boundary conditions (PBCs). Previous reduced physical domains of NETmix include the NETmix Unit Block (NUB) (Fonte et al., 2013; Costa et al., 2017) which will be revised in this work.

The simulation of the NETmix mixing dynamics in each chamber is affected by the boundary conditions. In this way, the reduced geometry needs to ensure that the model does not affect the mixing dynamics. This was thoroughly covered by Torres (2017) from spectral analysis. In this work, the reduced geometric model, ExtendedNUB, will be presented and validated from second-moment statistics. Turbulence intensity is related to the magnitude of the velocity fluctuation of the fluid associated with an enhancement of mixing in laminar chaotic or turbulent flows (Pope 2000).

The main operational parameter in NETmix for single phase flows is the Reynolds number of the channels, Re. The Reynolds number in the NETmix reactor is defined at the inlet channels as $\operatorname{Re}=\frac{\rho 2 d v_{i n j}}{\mu}$, in which $d$ is the width of the channels, $v_{i n j}$, is the velocity of the fluid at the inlets and $\rho$ and $\mu$ are the density and viscosity of the fluid, respectively. This parameter marks the transition from segregated laminar, where fluids flow segregated on their side of the chambers, to chaotic flow regimes (Laranjeira et al., 2011). Chaotic flow regimes occur above a critical Reynolds number of ca. 120 (depending on the network topology), when the impingement point of the inlet jets inside the mixing chambers starts oscillating and onsets a dynamic behaviour of the vortices (Laranjeira et al., 2009; Laranjeira et al., 2011). The engulfment of the vortices that are formed specially at the top and bottom of the chambers is responsible for mixing. In fact, tracer experiments have shown that above a certain row of chambers it is not possible to distinguish the flow patterns in the chambers due to the occurrence of a quasi-homogenization (Laranjeira et al., 2011).

Mixing at larger scales, i.e., macromixing, can be assessed in the NETmix reactor through the visualization of the overall patterns as shown in Figure 1, using tracer simulations which measure the relation between the composition of the fluids at the inlet and outlet. In each NETmix chamber, if the composition of the fluid at the outlet is diluted by a factor of two in relation to the composition of the fluid at the inlet of that chamber, perfect mixing occurs. The overall mixing capacity of the NETmix reactor is then $2^{n}$, in which $n$ represents the number of chambers. The composition of the fluid that leaves the NETmix network at the top row is diluted $2^{n}$ times in relation to the composition of the fluid that entered in the NETmix network at the bottom row. A way to evaluate the degree of mixing in the NETmix reactor is to use particles to monitor, at the outlet, the distribution of the fraction of the fluids being mixed. Considering one chamber, if the fraction of particles injected in the left inlet that exit the chamber through the right outlet is 0.5 , the dilution factor is 2 , and thus, mixing is perfect. In this work, the algorithm Batch Lagrangian Mixing Simulation (BLMS) was developed and implemented to access the mixing of two fluids in each mixing chamber.

The mixing capacity of reactors is highly dependent on the device topology. Since its conception, it was noticeable that the configuration of the network has a major impact on the NETmix performance (Laranjeira 2005; Gomes 2011). One of the first works was focused on the effect of the ratio between the volumes of the chambers and channels on micromixing. Laranjeira et al. (2009) used a network model where the chambers were modelled as perfectly mixing zones and the channels as plug flow 

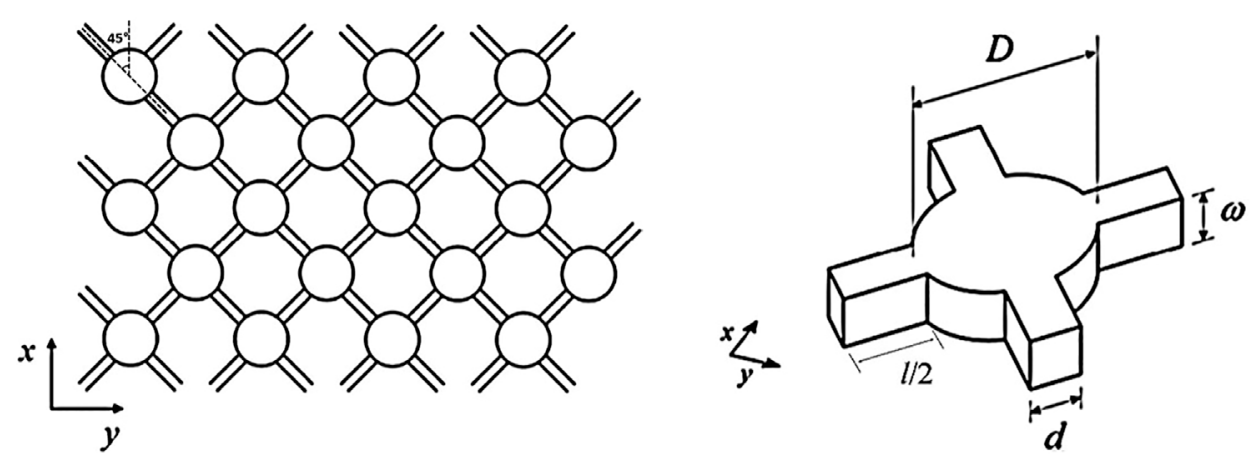

FIGURE 2 | Schematic representation of: NETmix network (A) and NETmix unit cell geometries (adapted from Fonte et al., 2013) (B)

segregation zones to study the NETmix network design. Results of the ratio between the volume of the channels and the whole network volume have led to the present NETmix configuration where each chamber is connected to four channels, forming an angle of $45^{\circ}$ with the chamber axis, i.e., $90^{\circ}$ angle between neighbouring channels. Previous works have also shown that macromixing and micromixing in NETmix depend on the number of chamber rows in the flow direction and on the injection scheme of the reactants (Laranjeira et al., 2009; Laranjeira et al., 2011).

Since the topology of the reactor exerts a major influence in the mixing dynamics of the NETmix chambers, this work is dedicated to the evaluation of mixing inside each chamber and, from this study, improve the design of the reactor. The NETmix topology will be optimised in terms of the ratio chamber diameter/channel width, $D / d$, to achieve a dilution factor as close as possible to two inside each mixing chamber. For that, BLMS will be applied in the ExtendedNUB model to analyse the influence that the ratio $D / d$ has in mixing.

\section{NETMIX NETWORK}

The NETmix model was studied and validated in previous works numerically and experimentally (Laranjeira et al., 2009; Laranjeira et al., 2011). The NETmix model uses a network composed of chambers interconnected by channels whose arrangement has a crucial impact on mixing.

The NETmix network (Figure 2 left) is composed of cylindrical chambers with a diameter, $D$, interconnected by parallelepipedic channels with width, $d$, and length, $l$, at a $45^{\circ}$ angle (Lopes et al., 2005; Laranjeira 2005; Gomes 2011). This network can be obtained by the repetition of the NETmix unit cell shown in Figure 2 (right), where $\omega$ is the reactor's depth. Experimental and computational results have shown that since mixing mechanisms are mainly $2 \mathrm{D}$ in the $x \mathrm{O} y$ plane (Gomes 2011), it is possible to accurately represent the mixing dynamics using a $2 \mathrm{D}$ model.

The mixing patterns from 2D CFD simulations are the same as those observed from tracer experiments, as will be later addressed in this work. The validity of the $2 \mathrm{D}$ modelling of the flow dynamics is restricted to the range of depth values tried so far,
$2 \leq \omega / d \leq 3.9$, which is close to a similar analysis on the validity of 2D assumption in T-jets reactors (Sultan et al., 2019). For the range of depth values that has been used for the design of NETmix reactors, the $2 \mathrm{D}$ physics provide an accurate description of the flow dynamics.

\section{ExtendedNUB Geometry}

Previous works have shown that the geometry and the flow in NETmix obey to a repetitive pattern (visible in Figure 1) which enables to simulate only a subdomain of the full network to study the NETmix reactor (Fonte et al., 2013; Costa et al., 2017). Fonte (2013) and Fonte et al. (2013) developed a 3D geometry that consisted of three full chambers interconnected to 6 half chambers, the NETmix Unit Block (NUB) shown in Figure 3 (left). The half chambers in each row of the network are connected to each other by a periodic boundary condition as shown in Figure 3 (left). Torres (2017) studied the possibility of simulating reduced NETmix geometries in $2 \mathrm{D}$ NUB models. One of the models, the ExtendedNUB, consisted in using a repetition of two NUBs (Figure 3 right). The ExtendedNUB geometry has two bottom inlets and two top outlets and comprises four full chambers (C2, C4, C6 and C8 in Figure 3 right) and five pairs of half chambers. This network has a total of nine rows of channels issuing from the chambers (nine left channels, $l 1$ to $l 9$ and nine right channels, $r 1$ to $r 9$ ). This geometry will be validated further in this work.

\section{CFD Model and Boundary Conditions}

The $2 \mathrm{D}$ domain was discretized with a symmetrical mesh in relation to the $x$ and $y$ axis, using ANSYS ICEM. A quarter of chamber is shown in Figure 4. The cell elements have an average edge length, $\Delta x=1.7 \times 10^{-5} \mathrm{~m}$.

The commercial finite volume CFD software ANSYS Fluent $^{\mathrm{TM}}$ was used to solve the mass and momentum conservation equations for the ExtendedNUB model, respectively defined as

$$
\begin{aligned}
\nabla \cdot \boldsymbol{v} & =0 \\
\rho\left(\frac{\partial \boldsymbol{v}}{\partial t}+\boldsymbol{v} \cdot \nabla \boldsymbol{v}\right) & =-\nabla p+\mu \nabla^{2} \mathbf{v}
\end{aligned}
$$



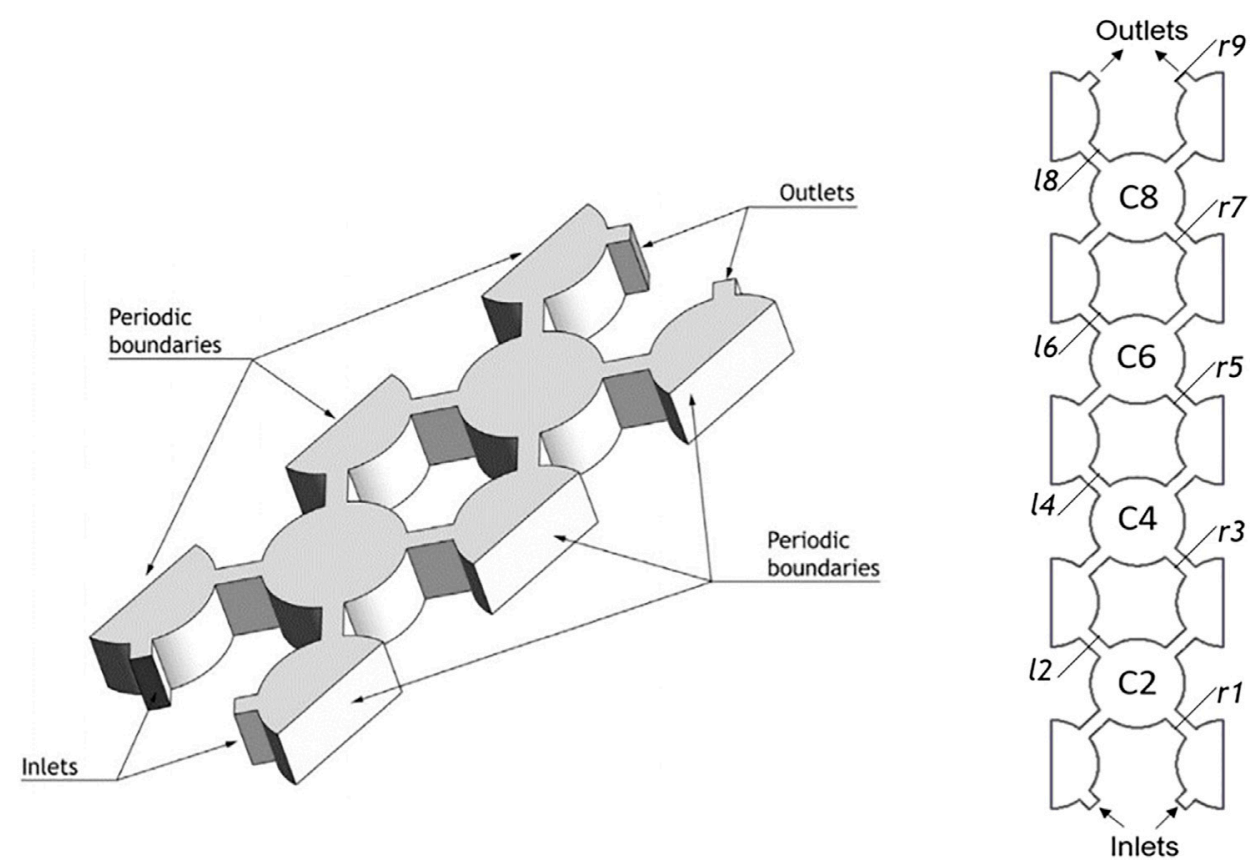

FIGURE 3 | Schematic representation of: NUB (Fonte et al., 2013) (left) and ExtendedNUB (with chambers 2, 4, 6, 8) (right).

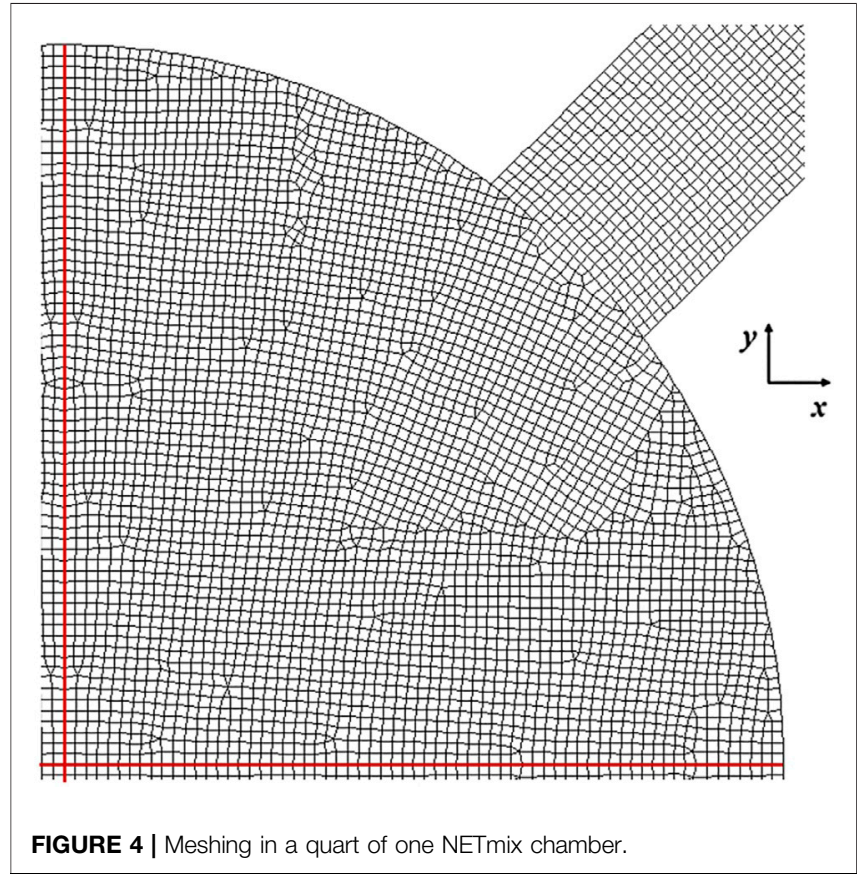

where $v$ is the velocity vector, $p$ is the pressure, $\rho$ and $\mu$ are the density and viscosity of the fluid, respectively. The SIMPLEC scheme was used with PRESTO! for the pressure-velocity coupling and Third-Order MUSCL for the spatial discretization. The convergence criterion for the maximum residuals of continuity and momentum is $10^{-5}$.
At the inlets, a constant velocity is imposed, $v_{i n j}$, which is obtained from the Reynolds number, according to

$$
v_{i n j}=\frac{\mu \mathrm{Re}}{\rho D_{h}}
$$

where $D_{h}=2 d$ is the hydraulic diameter for the $2 \mathrm{D}$ geometry, $\rho=998.2 \mathrm{~kg} / \mathrm{m}^{3}$ and $\mu=1.0 \mathrm{mPa}$.s.

At the outlets, a constant and uniform pressure profile is used. As extensively analysed by Torres (2017), it is possible to use reduced geometries with only one column to represent the NETmix network if periodic boundaries are used at the half chambers, as shown in Figure 3. A periodic boundary condition is then set at each pair of half lateral chambers. Velocity components $x$ and $y$ are set to zero at the walls of NETmix.

To perform the transient CFD simulation, the initial solution was obtained by setting all variables to zero.

\section{D ExtendedNUB Geometry Validation}

To validate the 2D ExtendedNUB model, the dynamic flow field was simulated for a geometry with a chamber diameter, $D=6.65 \times 10^{-3} \mathrm{~m}$, channel length, $l=2.0 \times 10^{-3} \mathrm{~m}$ and channel width, $d=1.0 \times 10^{-3} \mathrm{~m}$. Several Reynolds numbers were used from 100 to 340. The time step was calculated according to the Courant number, $\mathrm{C}=\frac{v_{i n} \Delta t}{\Delta x}=1$. As an example, for $\mathrm{Re}=300, \Delta t=$ $1.14 \times 10^{-4} \mathrm{~s}$.

The residence time in one NETmix chamber is given by $\tau_{c}=$ $\frac{\pi D^{2}}{8 v_{i n j} d}=0.12 \mathrm{~s}$ for the geometry dimensions above mentioned. The residence time of the entire ExtendedNUB is then $\tau=1.1 \mathrm{~s}$.

In CFD simulations, the oscillating behaviour referred in the Introduction and shown in Figure 1 takes about $9 \tau(10 \mathrm{~s}$ 


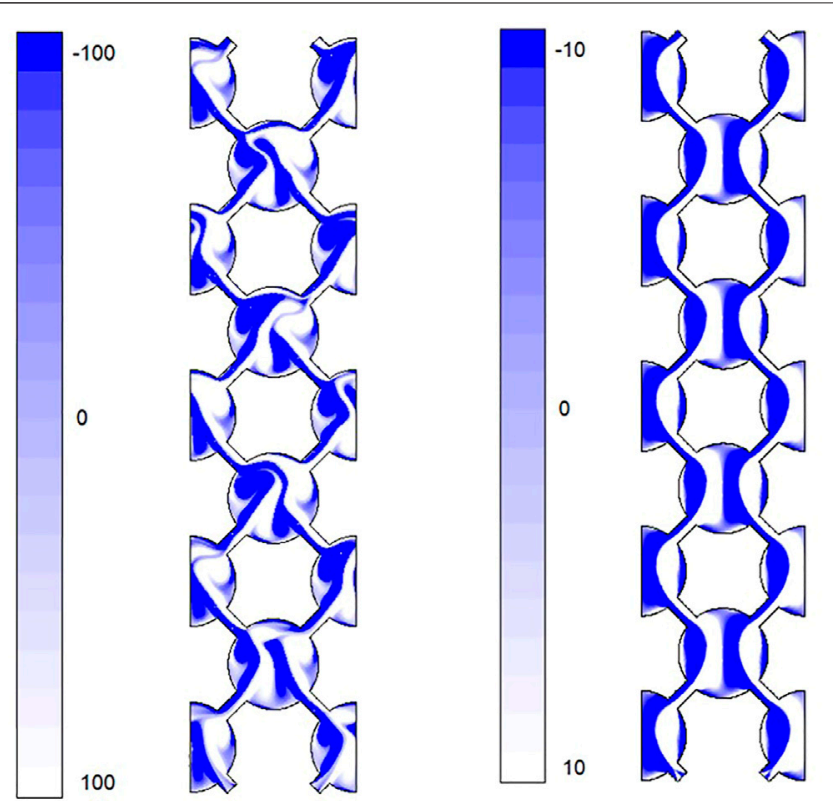

FIGURE 5 | Contour plot of the $z$-component of the vorticity of the $2 \mathrm{D}$ ExtendedNUB simulation at $34 \mathrm{~s}$ for $\mathrm{Re}=300$ (A) and $\mathrm{Re}=100$ (B).

flowtime) to be reached as will be shown further in this work. The simulations need, then, to be run for more than $10 \mathrm{~s}$ in order to generate dynamic data that represents the characteristic oscillating behaviour of the NETmix reactor. In this case, the simulation was run during $34 \mathrm{~s}$ flowtime and the results were recorded for analysis only from 17 to $34 \mathrm{~s}$. As an example, the contour plot of the $z$-component of the vorticity for the $2 \mathrm{D}$ ExtendedNUB simulation with $\mathrm{Re}=300$ is shown in Figure 5 (left), where the oscillating behaviour inside the NETmix chambers along with the vortex formation on the top and bottom of each chamber is observed. The vortices engulf the jets issuing from the channels and make the angle of contact between the two jets oscillate periodically from left to right. The overall mixing pattern is consistent with the one shown in the tracer simulation of Figure 1, which is also observed from experiments (Laranjeira et al., 2011). In contrast, if a Reynolds number below the critical value is chosen, the oscillating behaviour inside the chambers does not occur and there is no formation of vortices that dynamically evolve in the chamber, as shown in Figure 5 (right) for $\mathrm{Re}=100$.

When boundary conditions are imposed to a reduced model, it is necessary to ensure that the flow dynamics in the mixing chambers are not affected. As described in CFD Model and Boundary Conditions, at the inlet, there is a constant velocity and at the outlet, the constant and uniform pressure profile imposes that the velocity vectors are normal to the outlet surface, i.e., parallel outlet flow, which causes damping of the flow dynamics. In this way, turbulence intensity, $I_{v_{x}}$, studies were performed in the centre of all full chambers C2, C4, C6 and C8, in the direction of the main flow oscillations.

The turbulence intensity is defined by

$$
I_{v_{x}}=\frac{1}{v_{i n j}} \sqrt{\frac{\sum_{i=1}^{n} t_{i}\left(v_{x_{i}}-\overline{v_{x}}\right)^{2}}{\sum_{i=1}^{n} t_{i}}},
$$

where $v_{i n j}$ is the surface velocity at the inlet, $v_{x_{i}}$ is the $x$ component of the velocity at several flowtimes, $\overline{v_{x}}$ is the average of $v_{x_{i}}$ and $t_{i}$ is the flowtime.

The onset of chaotic mixing mechanisms in NETmix occurs above a critical Reynolds (ca. 120) where the steady flow evolves to fully developed laminar chaotic until $\operatorname{Re}=300$. For $\operatorname{Re}>300$, the head loss increases without significant improvement in the mixing performance (Fonte et al., 2013). To perform the turbulence intensity study, different Reynolds numbers were chosen from 140 (just above critical) to 300 , by varying the inlet velocity. The time-step size was also recalculated to maintain the Courant number at 1 .

The inlet velocity and time-step size of each case are: $v_{i n j}=7.02 \times 10^{-2} \mathrm{~m} / \mathrm{s}, \quad \Delta t=2.44 \times 10^{-4} s \quad$ for $\operatorname{Re}=140$; $v_{i n j}=9.03 \times 10^{-2} \mathrm{~m} / \mathrm{s}, \quad \Delta t=1.90 \times 10^{-4} \mathrm{~s}$ for $\operatorname{Re}=180$; $v_{i n=j} 1.10 \times 10^{-1} \mathrm{~m} / \mathrm{s}, \Delta t=1.55 \times 10^{-4} s$ for $\operatorname{Re}=220 ; v_{i n j}=1.31 \times$ $10^{-2} \mathrm{~m} / \mathrm{s}, \Delta t=1.31 \times 10^{-4} s$ for $\operatorname{Re}=260$; and $v_{i n j}=1.51 \times 10^{-2}$ $\mathrm{m} / \mathrm{s}, \Delta t=1.14 \times 10^{-4} \mathrm{~s}$ for $\mathrm{Re}=300$.

Figure 6 shows that the turbulent intensity is larger than zero for all Reynolds numbers. The flow is then above the critical $\mathrm{Re}$ which onsets dynamic flows. For lower Re, between 140 and 220, the turbulence intensity is nearly constant. As Re increases, the turbulence intensity is higher, as expected. At $\operatorname{Re}=260$, an increase is observed for chamber $\mathrm{C} 8$. At $\mathrm{Re}=300$ it is visible that the turbulence intensity is higher in chamber C6 and then decreases in chamber C8. This is explained by the effect of the outlet boundary condition, which dampens the oscillations. In this way, since this effect is not noticeable in chamber C6, the ExtendedNUB model can be used to represent the NETmix reactor for $\mathrm{Re}=300$ if the studies are performed in chamber C6.

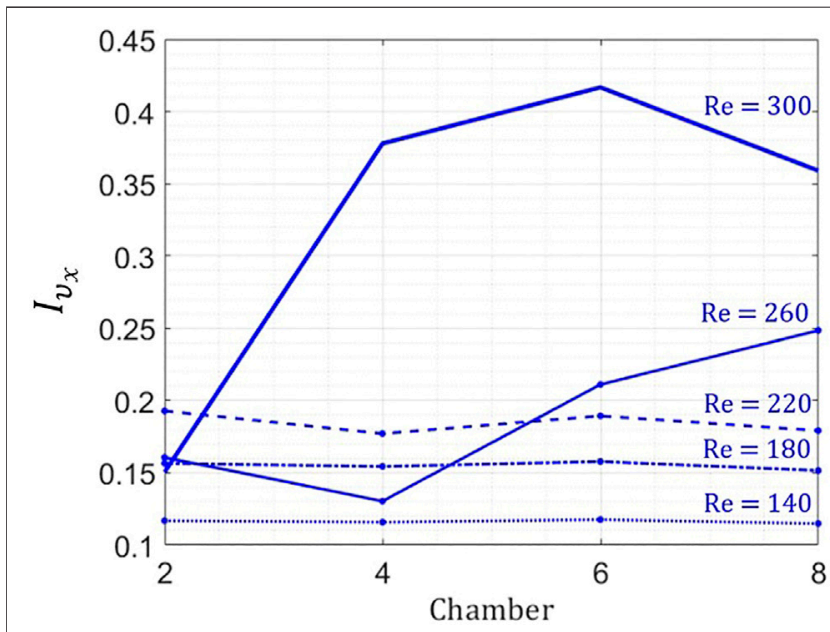

FIGURE 6 | Turbulence intensity on the centre of ExtendedNUB chambers C2, C4, C6 and C8 for D/d = 6.65 at Re = 140, 180, 220 and 260 and 300 , from 17 to $34 \mathrm{~s}$ flowtime. 


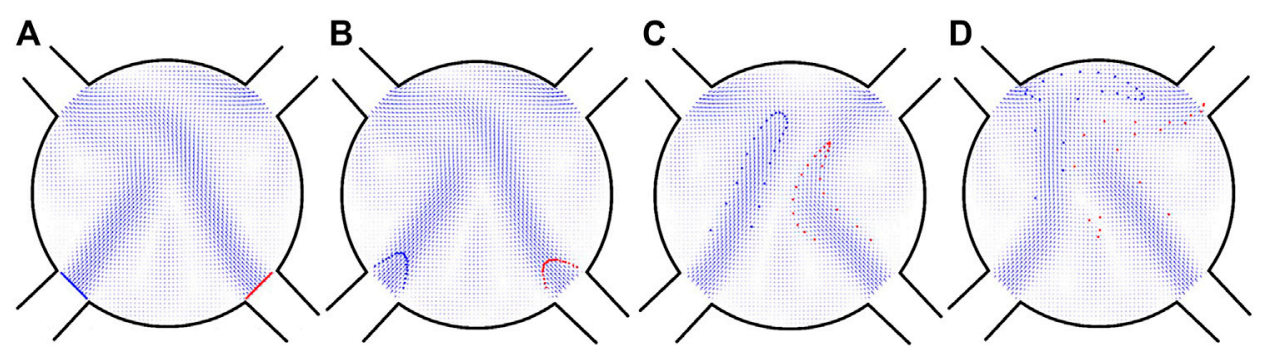

FIGURE 7 | Injection of particles over the velocity field in one NETmix chamber.

\section{NETMIX NETWORK TOPOLOGY OPTIMISATION}

In the NETmix reactor, the degree of mixing that occurs in one chamber can be analysed through the outlet distribution of the fluids being mixed, i.e., the fraction of fluid injected at the left inlet that goes out through the right outlet and vice-versa. In this work, Lagrangian methods will be used to track the injected fluids from the inlet to the outlet of one chamber. This algorithm will then be used to optimise the NETmix network topology in terms of the ratio chamber diameter/channel width, $D / d$, which is expected to have a major influence on the mixing capacity of the NETmix reactor.

\section{Batch Lagrangian Mixing Simulation (BLMS)}

Batch Lagrangian Mixing Simulations (BLMS) was based on the computational implementation of a previous algorithm, Lagrangian Mixing Simulation (LMS) presented by Matos et al. (2018). In LMS, a continuous injection of particles is used to track the filling front of fluids being mixed, according to

$$
\mathbf{X}_{i, j}=\mathbf{X}_{i-1, j}+10 \mathbf{v}_{i-1, j} \Delta t
$$

in which $\mathbf{X}_{i, j}$ is the matrix of the positions of the particles, $\Delta t$ is the time step (which is equal to the time step size used for the CFD simulation that provided the flow-field) and $v$ is a matrix with the velocities of the particles, $i$ represents the time (iteration) and $j$ the particle. The time difference between the velocity fields used for the tracking is $10 \Delta t$. In Eq. 5, the time interval is then set accordingly.

The position matrix of the particles, $\mathbf{X}_{i, j}$, is growing in LMS since new particles are inserted when two consecutive particles become apart more than $1.0 \times 10^{-4} \mathrm{~m}$. This step of inserting new particles is used to keep the accuracy of the fluid front tracking since the main objective of LMS is to determine all convective mixing scales.

The degree of mixing in the NETmix reactor can be assessed from macromixing in a unitary cell where the dynamic flow is fully developed. Since this can be done without simulating the full complexity of the interface between the fluids being mixed, independent discrete particles are used. In BLMS the number of particles is constant at each injection, which enables to follow the path of each particle from the inlet to the outlet and associate it to a fraction of each fluid being injected.

A first batch injection is set at flowtime $t_{0}$. Each injection is performed during $n i$ iterations until all the particles have left the mixing chamber. The process is then restarted and a new injection is set at $t_{0}+10 \Delta t$, in which $\Delta t$ is the time-step size of the CFD simulation that provided the velocity-fields. In this way, a series of injections are performed with interval $10 \Delta t$ until the total number of injections, $n t$, is reached. The injection procedure is represented in Figure 7, in which two series of $n p$ particles (the number of particles will be discussed further in this section) are injected, a red series at the right inlet and a blue series at the left inlet. The example of Figure 7 shows BLMS applied at chamber C6 of the 2D ExtendedNUB model with $D / d=$ 6.65 for $\operatorname{Re}=300$.

In Figure 7A-D, the velocity field vectors show oscillating behaviour inside the mixing chamber. The oscillations are also visible from the path of the particles, which follows the inlet streams (Figures 7B,C) and then are engulfed by the dynamic vortices that onset in NETmix above the critical Re (Figure 7D). In Figure 7D it is possible to see the particles leaving the mixing chamber, which enables to register the number of blue and red particles that exit the mixing chamber through the left or right outlet. The complete BLMS algorithm was developed in MATLAB and considers the following steps:

\section{Bach Lagrangian Mixing Simulation (BLMS) Algorithm}

1. Specify $t_{0}, n p, \Delta t, n i$ and $n t$;

2. Load the first velocity-field file, $i=1$, for the first injection, $n=1$, at $t_{0}+(n-1) 10 \Delta t$

3. Position the particles over the inlet;

4. Calculate the velocities of the particles with MATLAB function interp2();

5. Recalculate the position of the particles with the LMS equation (Eq. 5);

6. Register the number of blue and red particles that exit the mixing chamber on the left or right outlet, $n_{b}^{l}, n_{b}^{r}, n_{r}^{l}$ and $n_{r}^{r}$;

7. Load the next velocity field, $i+1$, at $t_{0}+i 10 \Delta t$;

8. Repeat steps 4 to 6 until $i=n i$;

9. Load the first velocity-field file, $i=1$, for the next injection, $n=n+1$, at $t_{0}+(n-1) 10 \Delta t$

10. Repeat steps 3 to 8 until $n=n t$. 


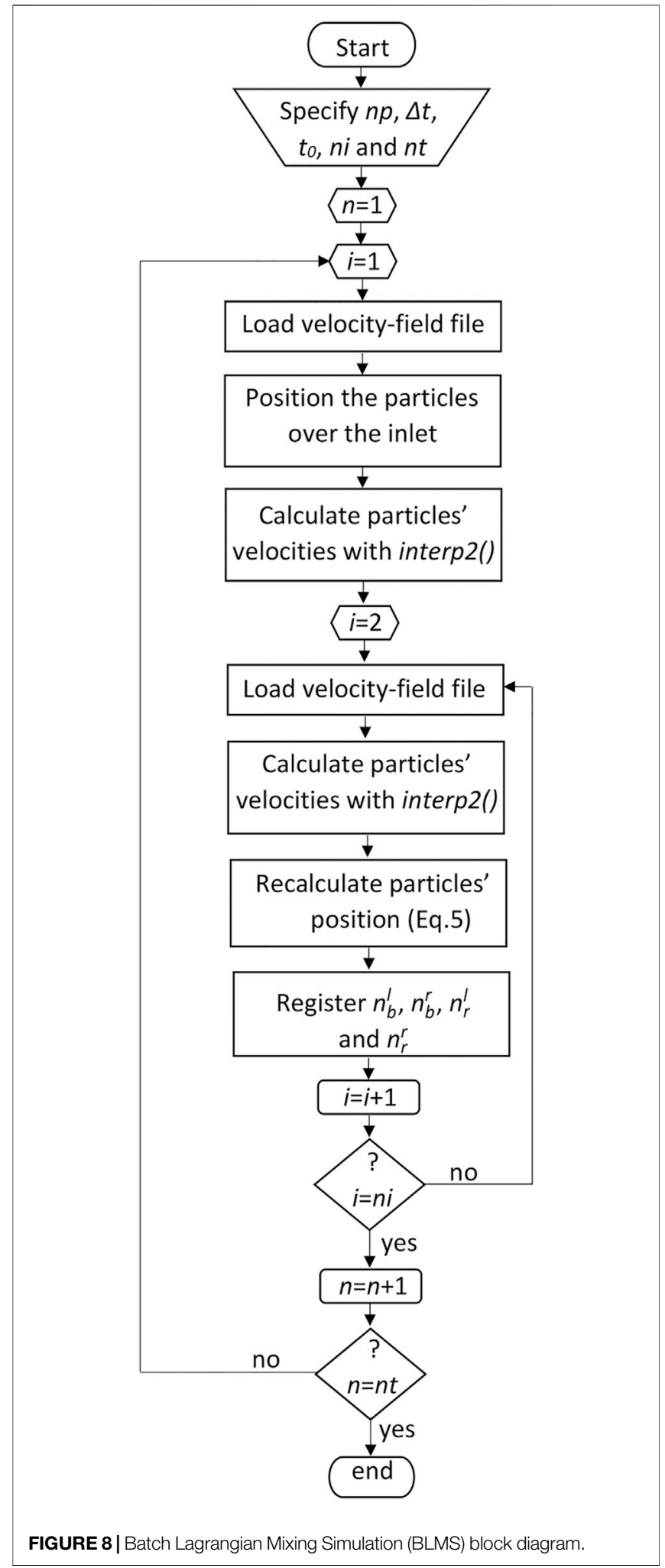

The respective block diagram is shown in Figure 8.

In BLMS, the position of the particles at the inlets was set according to the flow-field, the zones with higher velocity had a larger density of particles. i.e., the distance between the particles is

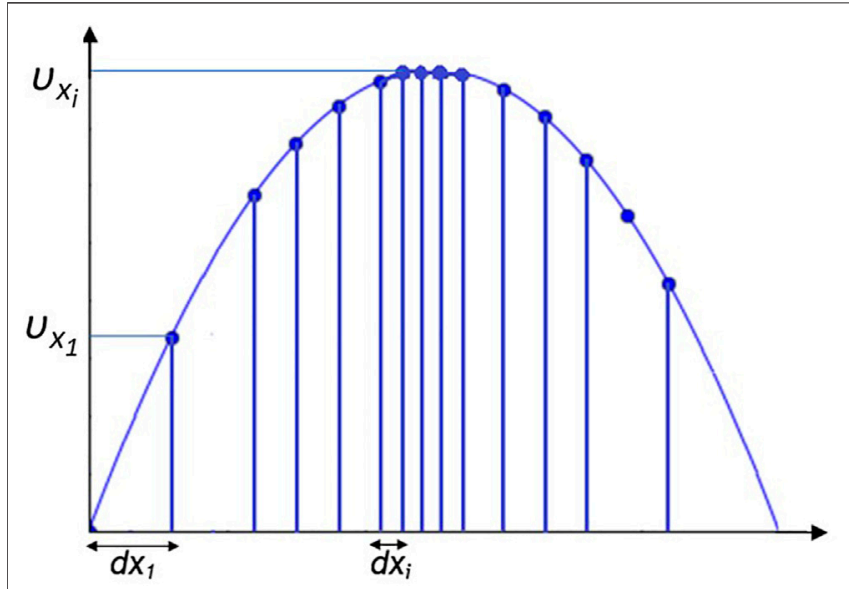

FIGURE. 9 | Scheme of the calculation procedure of $d x_{i}$.

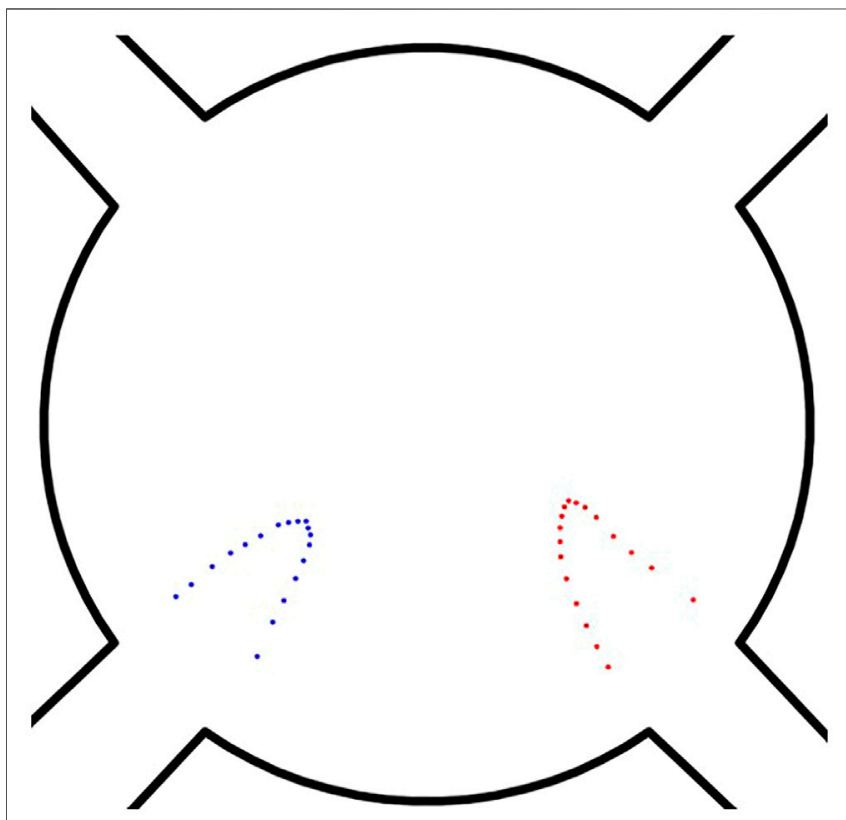

FIGURE 10 | Particle's injection profile in one NETmix chamber.

smaller. The velocity profile, $v_{x_{i}}$, at the inlet is close to a parabolic profile. The distance between two consecutive particles, $d_{x_{i}}$, was than calculated in order to have $d_{x_{i}}\left(v_{x_{i}}-v_{x_{i}-1}\right)=$ constant. A schematic representation is in Figure 9 and the algorithm for the calculation of the distance between the particles, $d x_{i}$ is presented next.

\section{Particles Distribution Over the Inlet Algorithm}

1. Select the coordinates $x$ and $y$ for the first and last particles $(P 1$ and $P n$ ), according to the coordinates of the inlet channel;

2. Draw a line between $P 1$ and $P n$;

3. Build vectors $\mathrm{x} 0$ and $v 0$ with $x$-coordinate and $x$-velocity of the mesh cells that are crossed by the line in 2 ; 


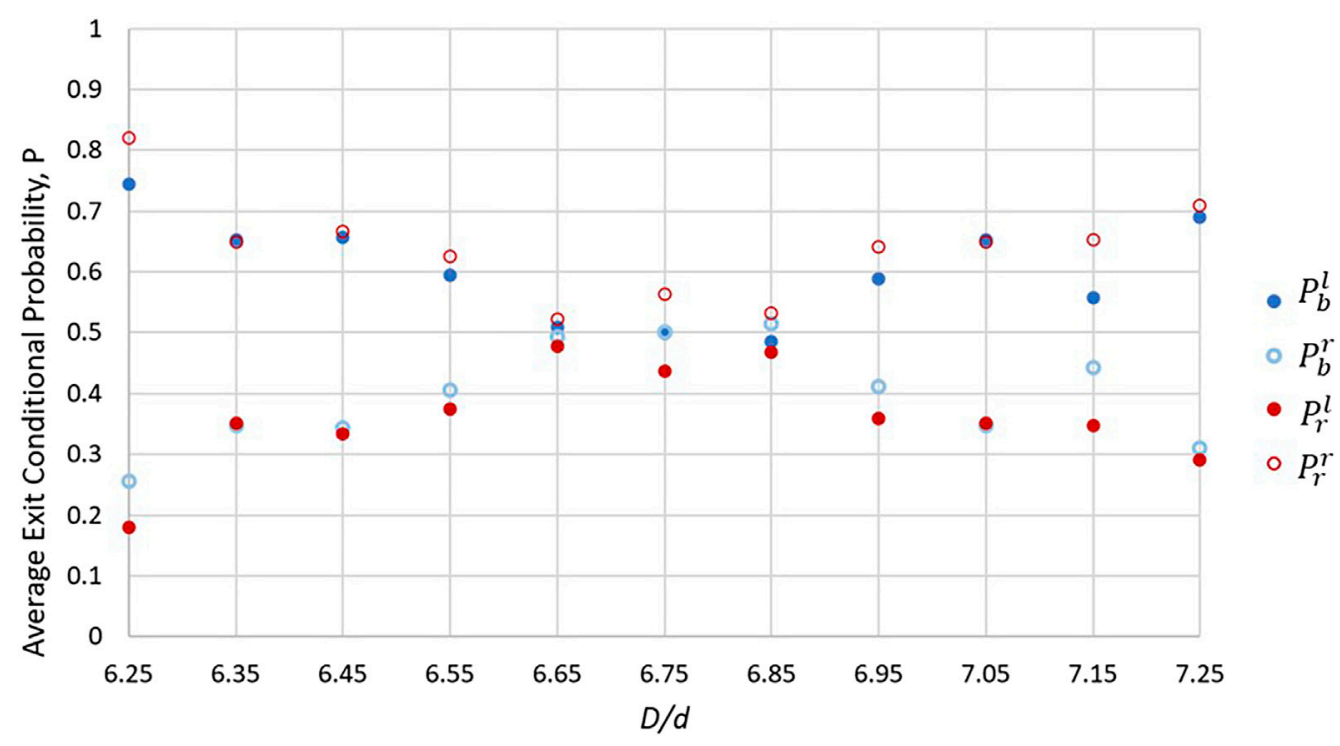

FIGURE 11 | Average of the exit conditional probability for $D / d$ from 6.25 to 7.25 in chamber C6 of 2D ExtendedNUB model for Re $=300$.

4. Start with $d x_{i}=2.5 \times 10^{-5} \mathrm{~m}$;

5. Calculate the area below $v_{x}$ curve: $A_{i}=\frac{v_{x_{i}}+v_{x_{i-1}}}{2} d x_{i}$;

6. Calculate the difference between consecutive areas: $\Delta A=\left|A_{i}-A_{i-1}\right|$;

7. Reduce $d x_{i}$ by a factor of $10^{-7} \mathrm{~m}$ and repeat steps 5 and 6 until $\Delta A \leq 10^{-8} \mathrm{~m}^{2} / \mathrm{s}$.

8. Repeat steps 4 to 7 until the coordinates of $P n$ are reached.

9. The number of intervals, $i$, that are needed to reach $P n$ is used to calculate the number of particles of each series, $n p=i+1$.

Figure 10 shows the beginning of the particle tracking after one injection where it is visible that in the tip of the profile the density of particles is larger.

\section{Diameter Ratio Study}

The ratio chamber diameter/channel width, $D / d$, is the critical dimension for mixing in NETmix, when the depth is in the range of depth values where the $2 \mathrm{D}$ assumption is valid. BLMS was applied in the 2D ExtendedNUB model for chamber diameters in the range $6.25 \times 10^{-3} \mathrm{~m} \geq D \geq 7.25 \times 10^{-3} \mathrm{~m}$; the channel length, $l=2.0 \times 10^{-3} \mathrm{~m}$ and the channel width, $d=$ $1.0 \times 10^{-3} \mathrm{~m}$ were kept constant. For each $D / d$, a CFD simulation, similar to the one shown in Figure 5 (left), was performed for $\mathrm{Re}=300$ to provide the velocity field for the injection of particles with BLMS. For each $D / d$ the velocity field data of chamber C6 was used to perform $n t=5000$ injections, each one during $0.58 \mathrm{~s}$ flowtime ( $n i=510$ iterations). Note that, since each NETmix chamber has a residence time, $\tau=0.12 \mathrm{~s}$ (for $D / d=6.65)$, the particles are tracked for ca. $3 \tau$. The first injection was made at $t_{0}=17 \mathrm{~s}$ flowtime. The number of particles for each series depends on the ratio $D / d$ and varied from 25 to 35 .

To evaluate the particles distribution at the outlet, the exit conditional probabilities were computed. $P_{b}^{l}$ is the probability of a blue particle exiting the mixing chamber on the left outlet, $P_{b}^{r}$ is the probability of a blue particle exiting the mixing chamber on the right outlet, $P_{r}^{l}$ is the probability of a red particle exiting the mixing chamber on the left outlet and $P_{r}^{r}$ is the probability of a red particle exiting the mixing chamber on the right outlet. These probabilities were calculated according to

$$
\begin{aligned}
& P_{b}^{l}=\frac{n_{b}^{l}}{n_{b}^{l}+n_{b}^{r}}, \\
& P_{b}^{r}=\frac{n_{b}^{r}}{n_{b}^{l}+n_{b}^{r}} \\
& P_{r}^{l}=\frac{n_{r}^{l}}{n_{r}^{l}+n_{r}^{r}}, \\
& P_{r}^{r}=\frac{n_{r}^{r}}{n_{r}^{l}+n_{r}^{r}}
\end{aligned}
$$

in which $n_{b}^{l}$ is the number of blue particles that exit on the left outlet, $n_{b}^{r}$ is the number of blue particles that exit on the right outlet, $n_{r}^{l}$ is the number of red particles that exit on the left outlet and $n_{r}^{r}$ is the number of red particles that exit on the right outlet (see point six of the BLMS algorithm).

The results presented in Figure $\mathbf{1 1}$ show that the relation between macromixing in the NETmix chambers and the ratio $D /$ $d$ is represented by a butterfly shaped graphic centred at $D / d=$ 6.75. For all ratios, the plot is symmetrical in relation to the probability line, $P=0.5$. For both the red and blue particles, the ratios 6.65 and 6.85 are the ones that have the best mixing since the exit conditional probability is very close to 0.5 which indicates that the dilution factor of two is attained. Looking to all the ratios, 6.75 is the one that has a probability closest to 0.5 ; however, this only happens for the blue particles. As the ratios move from the middle value 6.75 to the edges, 6.25 and 


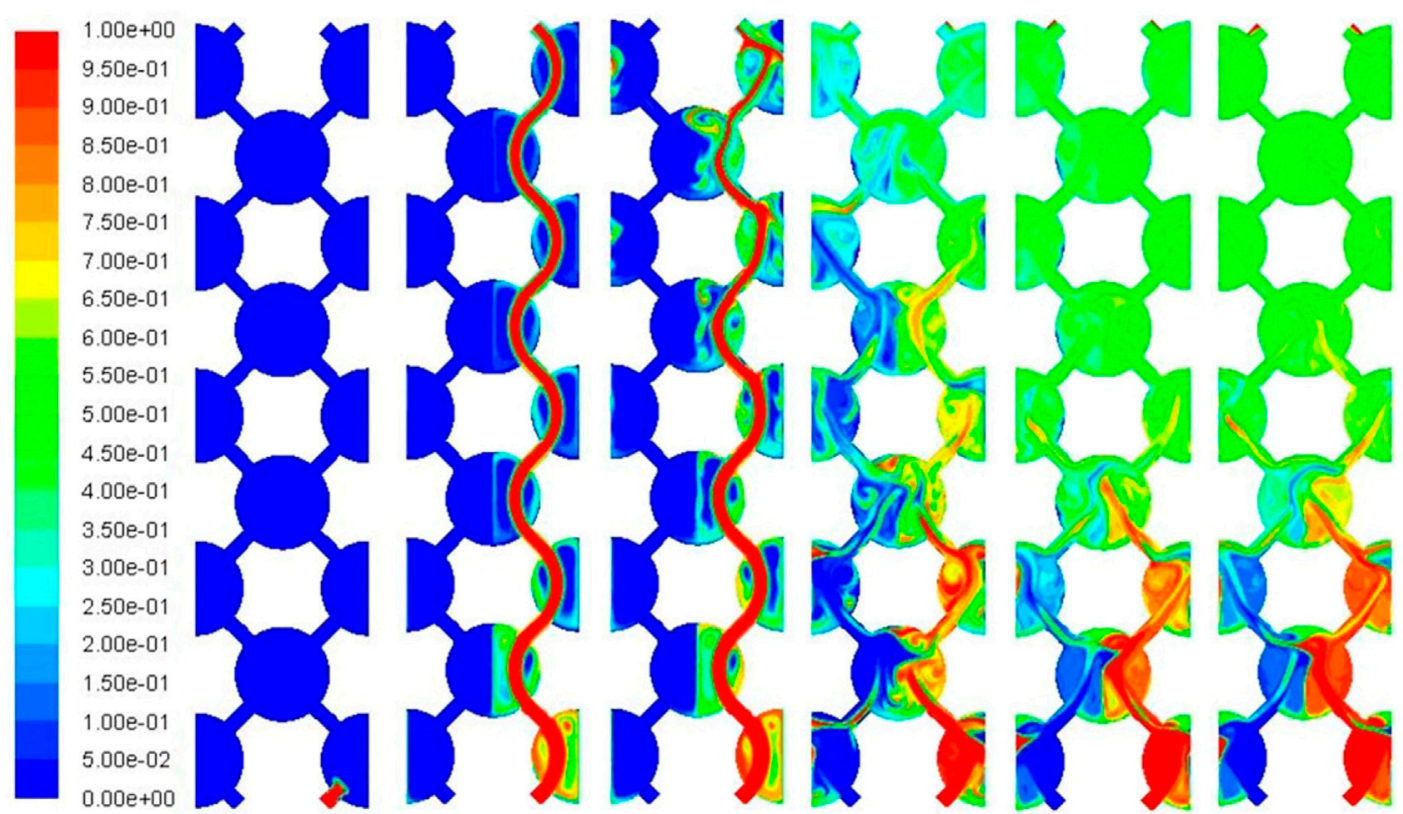

FIGURE 12 | Molar fraction of Fluid2 in the ExtendedNUB geometry, for Re= 300 , at: (A) 0.034 s, (B) 0.94 s, (C) 1.95 s, (D) 2.63 s, (E) 3.52 s and (F) 5.69 s.

7.25, the probability moves away from 0.5 and the mixing rate decreases. $D / d=6.65$ is the ratio which has the overall average, i.e., the average for both, the blue and the red sets of particles, closest to 0.5 , so this ratio is considered the optimal geometry for unitary cells.

\section{TRACER SIMULATION}

In the previous section it was concluded that the ratio $D / d=6.65$ is the one that has better mixing. The ExtendedNUB geometry with this ratio is then going to be used to perform a tracer simulation. The tracer simulation will be used to assess the performance of the BLMS method to characterize mixing and validate the ExtendedNUB model for the simulation of mixing in NETmix.

The commercial finite volume CFD software ANSYS Fluent ${ }^{\mathrm{TM}}$ was used for the tracer simulation, considering the models described in CFD Model and Boundary Conditions, with the additional species transport equation

$$
\rho\left(\frac{\partial Y_{i}}{\partial t}+\nabla \mathbf{v} Y_{i}\right)=-\nabla \cdot \mathbf{J}_{i},
$$

in which $Y_{i}$ is the mass fraction of each species, $i$, and $\mathbf{J}_{\mathbf{i}}$ is the diffusion flux vector, defined as

$$
\mathbf{J}_{\mathbf{i}}=-\rho D_{i} \nabla Y_{i},
$$

in which $D_{i}=10^{-9} \mathrm{~m}^{2} / \mathrm{s}$ is the mass diffusion coefficient of species $i$ in the mixture. The spatial discretization of the geometry is also described in CFD Model and Boundary Conditions.

The tracer simulation was performed for $\mathrm{Re}=300$ and the parameters of $2 D$ ExtendedNUB Geometry Validation were maintained. Two fluids, Fluid1 and Fluid2, with the same properties of water were used. The reactor was initially filled with Fluid1. Fluid2 was injected through the right inlet when the dynamic simulation was started. Figure 12 shows the evolution of the molar fraction of Fluid2.

Figure 12A shows the injection of Fluid 2 at the right inlet. In Figure 12B, Fluid 2 is only present in the right side of the reactor since the oscillating behaviour of the jets formed in the NETmix channels is still being developed. In less than $2 \mathrm{~s}$, mixing starts to occur and Fluid 2 appears in the left side of the reactor (Figure 12C). The mixing process continues and in less than $6 \mathrm{~s}$, the average of the molar fraction of Fluid2 in the top chambers, C6 and C8 is already close to 0.5, as shown in Figure12F. Figure 13 (left) shows the history of the molar fraction of Fluid 2 in the centre of chamber C6 where from approximately $6 \mathrm{~s}$ flowtime the molar fraction of Fluid2 tends to 0.5 . The first $5 \mathrm{~s}$ correspond to the injection of Fluid2, which starts to occupy the reactor already filled with Fluid1. Although it takes just $6 \mathrm{~s}$ to have nearly perfect mixing inside the NETmix reactor, the oscillatory behaviour takes about $10 \mathrm{~s}$ to completely develop, as shown in Figure $\mathbf{1 3}$ (right) where only from $10 \mathrm{~s}$, the amplitude and frequency of the flow oscillations become constant.

In order to understand the mixing process of Fluid1 and Fluid 2 inside the reactor, the average of the molar fractions between 10 and $17 \mathrm{~s}$ flowtime was evaluated in the centre of each channel at the right, $r$, and at the left, $l$ (see Figure 3 right). The result is shown in Figure 14 where the fifth row of chambers is close to 0.5 . From the fifth row of chambers, the average of the molar fractions continues to approach 0.5 , reaching exactly these values in row 8 which are maintained in row 9.

This result shows that the ExtendedNUB geometry is capable of representing the behaviour of a larger NETmix network since 

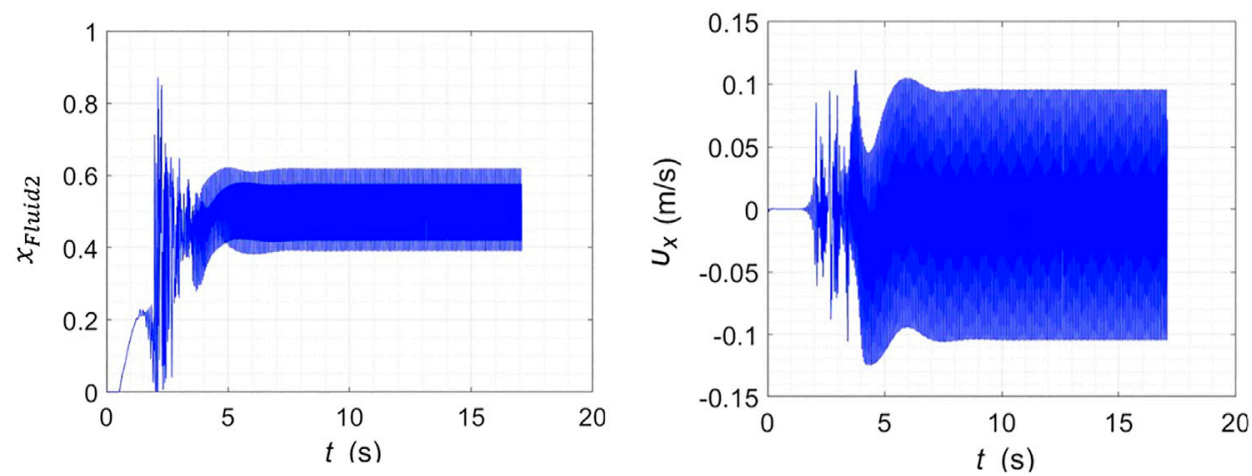

FIGURE 13 | Molar fraction history of Fluid2 (A) and $x$-velocity history (B) in the centre of chamber C6 of the ExtendedNUB geometry, for Re = 300.

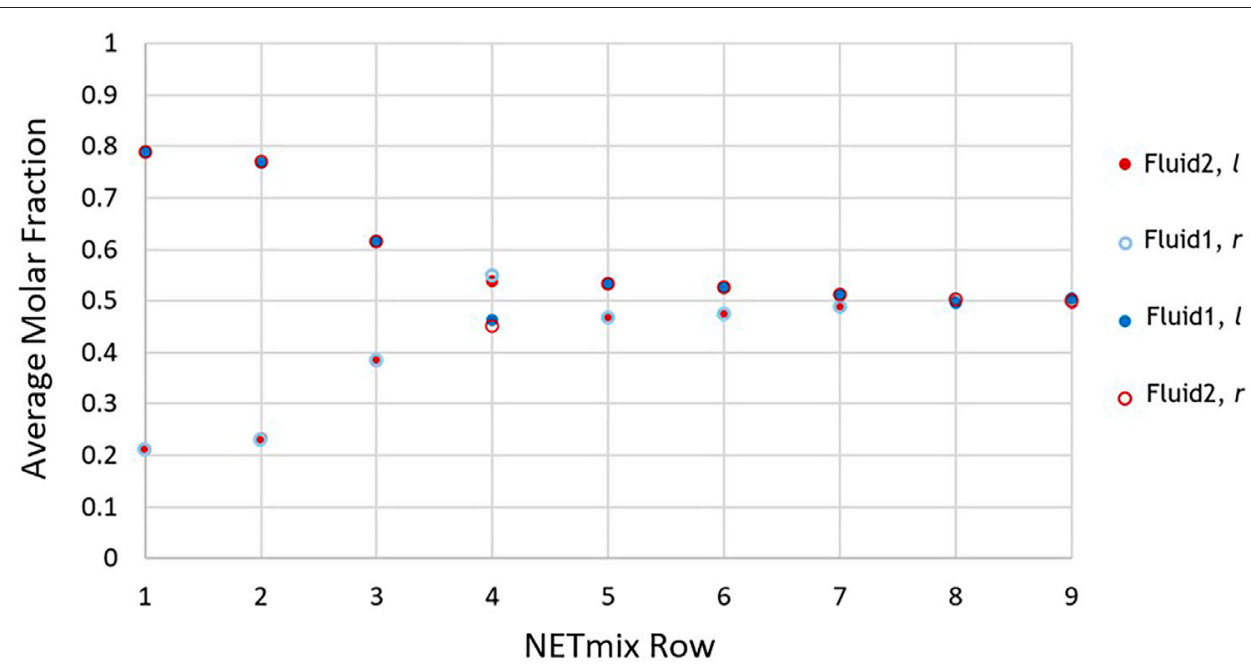

FIGURE 14 |Average of the molar fraction of Fluid1 and Fluid 2 in the centre of left and right channels of the ExtendedNUB geometry, for Re $=300$ between 10 and 17 s flowtime.

TABLE 1 | Comparison between BLMS and Tracer in chamber C6

\begin{tabular}{ccccc}
\hline & $P_{r}^{r}$ /Fluid2, right & $P_{r}^{\prime}$ /Fluid2, left & $P_{b}^{l}$ /Fluid1, left & $P_{b}^{r} /$ Fluid1, right \\
\hline BLMS & 0.52 & 0.48 & 0.51 & 0.49 \\
Tracer & 0.53 & 0.47 & 0.53 & 0.47
\end{tabular}

perfect mixing is reached in the top chambers. Moreover, nearly perfect mixing is obtained from the fifth row, i.e., before chamber C6.

The mixing patterns obtained from tracer simulations accurately described those obtained experimentally in geometries with depths in the range: $2 \leq \omega / d \leq 3.9$. This validates the 2D ExtendedNUB for simulation of flow and mixing in NETmix reactors in the range of depth values that are most typical in industrial applications. Moreover, this study was performed for single-phase mixing without heat exchange. For multiphase flows as well as reactive systems, especially if they include temperature variations, mixing features might change.

In the centre of chamber $\mathrm{C} 6$, the average of the molar fraction is nearly the same as the one reported by the BLMS study in $2 D$ ExtendedNUB Geometry Validation. Table 1 shows the comparison of the values for Fluid2 which was injected in the right side, represented in red, with the red particles that were injected in BLMS, $P_{r}^{l}$ and $P_{r}^{r}$. For Fluid1, which was injected in the left side, represented in blue, the comparison is performed with the blue particles of BLMS, $P_{b}^{l}$ and $P_{b}^{r}$. The similarity of the values between BLMS and the tracer simulation shows that the injection of particles is a good method to evaluate the performance of this type of reactor. 


\section{CONCLUSION}

For the first time, the degree of mixing of the NETmix chambers was assessed and the ratio chamber diameter/channel width, D/d, was studied. Mixing simulations show that it is possible to have nearly perfect mixing in the NETmix reactor for geometries in which the unit cell has $6.65 \geq D / d \geq 6.85$. The design of the unit cells of the NETmix network has then a major impact in the performance of the reactor. To characterize macromixing and evaluate the degree of mixing of the NETmix chambers, Batch Lagrangian Mixing Simulations (BLMS) were performed. The results have shown that for $6.65 \geq D / d \geq 6.85$ the average of the exit conditional probability is close to 0.5 . This indicates that a dilution factor of two is obtained between the inlet and outlet of the NETmix chambers, under fully developed flow conditions above the critical Reynolds number. A tracer simulation was performed for the best ratio, $D / d=6.65$, which shows that nearly perfect mixing is obtained within five rows of chambers, and perfect mixing is obtained with eight or more rows. A new reduced geometric model to represent the NETmix reactor, ExtendedNUB, was presented and validated. It was shown that the ExtendedNUB model simulates the hydrodynamics in the NETmix network without being affected by the inlet and outlet boundary conditions imposed on CFD.

\section{REFERENCES}

Costa, M. F., Fonte, C. M., Dias, M. M., and Lopes, J. C. B. (2017). Heat Transfer Performance of NETmix-A Novel Micro-meso Structured Mixer and Reactor. Aiche J. 63 (6), 2496-2508. doi:10.1002/aic.15728

Costa, M. F. (2017). The NETmix ${ }^{\circledR}$ Technology, Applied to Gas Hydrates Production: A Potential Solution to CCS. Porto: PhD Thesis of University of Porto.

da Costa Filho, B. M., Silva, G. V., Boaventura, R. A. R., Dias, M. M., Lopes, J. C. B., and Vilar, V. J. P. (2019). Ozonation and Ozone-Enhanced Photocatalysis for VOC Removal from Air Streams: Process Optimization, Synergy and Mechanism Assessment. Sci. Total Environ. 687, 1357-1368. doi:10.1016/ j.scitotenv.2019.05.365

Fonte, C. M., Leblebici, M. E., Dias, M. M., and Lopes, J. C. B. (2013). The NETmix Reactor: Pressure Drop Measurements and 3D CFD Modeling. Chem. Eng. Res. Des. 91 (11), 2250-2258. doi:10.1016/j.cherd.2013.07.014

Fonte, C. M. (2013). The NETmix Reactor: Application to High Added-Value Products. Porto: PhD Thesis of University of Porto.

Gomes, P. J., Silva, V. M. T. M., Quadros, P. A., Dias, M. M., and Lopes, J. C. B. (2009). A Highly Reproducible Continuous Process for Hydroxyapatite Nanoparticles Synthesis. J. Nanosci. Nanotech. 9 (6), 3387-3395. doi:10.1166/jnn.2009.ns06

Gomes, P. J. (2011). The NETmix ${ }^{\circledR}$ Reactor: Strategies for Optimizing Mixing and Development of New Reactor Designs. Porto: PhD Thesis of University of Porto.

Laranjeira, P. E., Martins, A. A., Lopes, J. C. B., and Dias, M. M. (2009). NETmix, A New Type of Static Mixer: Modeling, Simulation, Macromixing, and Micromixing Characterization. Aiche J. 55 (9), 2226-2243. doi:10.1002/ aic. 11815

Laranjeira, P. E., Martins, A. A., Nunes, M. I., Lopes, J. C. B., and Dias, M. M. (2011). NETmix, a New Type of Static Mixer: Experimental Characterization and Model Validation. Aiche J. 57 (4), 1020-1032. doi:10.1002/aic.12316

Laranjeira, P. E. (2005). NETmix Static Mixer, Modelling, CFD Simulation and Experimental Characterization. Porto: PhD Thesis of University of Porto.

\section{DATA AVAILABILITY STATEMENT}

The original contributions presented in the study are included in the article/Supplementary Material, further inquiries can be directed to the corresponding author.

\section{AUTHOR CONTRIBUTIONS}

JM and JL contributed to conception and design of the study. JM performed the simulations and analysis of the data and wrote the article. $\mathrm{MD}$ and RS revised the intellectual content. All authors contributed to article revision, read, and approved the submitted version.

\section{FUNDING}

This work was financially supported by: Base Funding - UIDB/ 50020/2020 and Programmatic-UIDP/50020/2020 Funding of LSRE-LCM, funded by national funds through FCT/MCTES (PIDDAC) and POCI-01-0145-FEDER-030445 - funded by European Regional Development Fund (ERDF) - Programa Operacional Competitividade e Internacionalização (POCI) and by national funds through FCT - Fundação para a Ciência e a Tecnologia IP; JM acknowledges her FCT scholarship SFRH/ $\mathrm{BD} / 137094 / 2018$.

Lima, M. J., Silva, A. M. T., Silva, C. G., Faria, J. L., Lopes, J. C. B., and Dias, M. M. (2016). An Innovative Static Mixer Photoreactor: Proof of Concept. Chem. Eng. J. 287, 419-424. doi:10.1016/j.cej.2015.09.092

Lopes, J. C. B., Dias, M. M. G. Q., Costa, M. F. S., Santos, R. J. N., and Teixeira, C. A. M. (2019). Continuous Production of Clathrate Hydrates from Aqueous and Hydrate-Forming Streams, Methods and Uses. Provisional European Patent Application 19220274.5. Submitted by PETROGAL, S.A.

Lopes, J. C. B., Dias, M. M. G. Q., Costa, M. F. S., Santos, R. J. N., and Teixeira, C. A. M. (2018). Network Heat Exchanger Device, Method and Uses. International Application PCT/IB2018/052463. Submitted by PETROGAL, S.A.

Lopes, J. C. B., Dias, M. M. Q., Silva, V. M. T. M., Santos, P. A. Q. O., Monteiro, F. J. M., Gomes, P. J. C., et al. (2006). Método de produção de nanopartículas de fosfatos de cálcio com elevada pureza e respetiva utilização. National Application PT 103528.

Lopes, J. C. B., Dias, M. M. Q., Silva, V. M. T. M., Santos, P. A. Q. O., Monteiro, F. J. M., Gomes, P. J. C., et al. (2007). Production Method for Calcium Phosphate Nano-Particles with High Purity and Their Use. International Application PCT/PT2007/000031.

Lopes, J. C. B., Laranjeira, P. E., Dias, M. M., and Martins. Network, A. A. (2005). Mixer and Related Mixing Process. in European Patent EP172643 B1. 2008, 2013, PCT/IB2005/000647, US Patent 8434933 B2.

Marinho, B. A., Djellabi, R., Cristóvão, R. O., Loureiro, J. M., Boaventura, R. A. R., Dias, M. M., et al. (2017). Intensification of Heterogeneous TiO2 Photocatalysis Using an Innovative Micro-meso-structured-reactor for $\mathrm{Cr}(\mathrm{VI})$ Reduction under Simulated Solar Light. Chem. Eng. J. 318, 76-88. doi:10.1016/ j.cej.2016.05.077

Matos, J., Brito, M. S. C. A., Dias, M. M., Lopes, J. C. B., and Santos, R. J. (2018). Lagrangian Mixing Simulation and Quantification of Scales. Chem. Eng. Sci. 192, 199-210. doi:10.1016/j.ces.2018.07.035

Moreira, A. C. G., Manrique, Y. A., Martins, I. M., Fernandes, I. P., Rodrigues, A. E., Lopes, J. C. B., et al. (2020). Continuous Production of MelamineFormaldehyde Microcapsules Using a Mesostructured Reactor. Ind. Eng. Chem. Res. 59 (41), 18510-18519. doi:10.1021/acs.iecr.0c02656

Pope, S. B. (2000). Turbulent Flows. Cambridge, UK: Cambridge University Pres. 
Ribeiro, A., Manrique, Y. A., Barreiro, F., Lopes, J. C. B., and Dias, M. M. (2021). Continuous Production of Hydroxyapatite Pickering Emulsions Using a Mesostructured Reactor. Colloids Surf. A: Physicochemical Eng. Aspects 616, 126365. doi:10.1016/j.colsurfa.2021.126365

Saber, M., Commenge, J. M., and Falk, L. (2010). Microreactor Numbering-Up in Multi-Scale Networks for Industrial-Scale Applications: Impact of Flow Maldistribution on the Reactor Performances. Chem. Eng. Sci. 65 (1), 372-379. doi:10.1016/j.ces.2009.06.010

Santos, S. G. S., Paulista, L. O., Marinho, B. A., Passalía, C., Flores, M., Labas, M. D., et al. (2021). A Step Forward on NETmix Reactor for Heterogeneous Photocatalysis: Kinetic Modeling of As(III) Oxidation. Chem. Eng. J. 405, 126612. doi:10.1016/j.cej.2020.126612

Santos, S. G. S., Paulista, L. O., Silva, T. F. C. V., Dias, M. M., Lopes, J. C. B., Boaventura, R. A. R., et al. (2019). Intensifying Heterogeneous $\mathrm{TiO} 2$ Photocatalysis for Bromate Reduction Using the NETmix Photoreactor. Sci. Total Environ. 664, 805-816. doi:10.1016/j.scitotenv.2019.02.045

Schenk, R., Hessel, V., Hofmann, C., Kiss, J., Löwe, H., and Ziogas, A. (2004). Numbering-up of Micro Devices: a First Liquid-Flow Splitting Unit. Chem. Eng. J. 101 (1-3), 421-429. doi:10.1016/j.cej.2003.11.034

Silva, V. M. T. M., Quadros, P. A., Laranjeira, P. E. M. S. C., Dias, M. M., and Lopes, J. C. B. (2008). A Novel Continuous Industrial Process for Producing Hydroxyapatite Nanoparticles. J. Dispersion Sci. Technol. 29, 542-547. doi:10.1080/01932690701728924
Sultan, M. A., Pardilhó, S. L., Brito, M. S. C. A., Fonte, C. P., Dias, M. M., Lopes, J. C. B., et al. (2019). 3D Mixing Dynamics in T-Jet Mixers. Chem. Eng. Technol. 42 (1), 119-128. doi:10.1002/ceat.201700684

Torres, J. P. (2017). Proper Orthogonal Decomposition of Turbulent Flows. Porto: MSc Thesis of University of Porto.

Conflict of Interest: The authors declare that the research was conducted in the absence of any commercial or financial relationships that could be construed as a potential conflict of interest.

Publisher's Note: All claims expressed in this article are solely those of the authors and do not necessarily represent those of their affiliated organizations, or those of the publisher, the editors and the reviewers. Any product that may be evaluated in this article, or claim that may be made by its manufacturer, is not guaranteed or endorsed by the publisher.

Copyright (c) 2021 Matos, Santos, Dias and Lopes. This is an open-access article distributed under the terms of the Creative Commons Attribution License (CC BY). The use, distribution or reproduction in other forums is permitted, provided the original author(s) and the copyright owner(s) are credited and that the original publication in this journal is cited, in accordance with accepted academic practice. No use, distribution or reproduction is permitted which does not comply with these terms. 


\section{NOMENCLATURE}

\section{SYMBOLS}

$A_{i}[\mathrm{~m} 2]$ Area below vx curve

$C$ [ ] Courant number

$d[\mathrm{~m}]$ NETmix channel width

$D[\mathrm{~m}]$ NETmix chamber diameter

D/d [ ] ratio Chamber diameter/Channel width

$D_{h}[\mathrm{~m}]$ hydraulic diameter

$d x_{i}[\mathrm{~m}]$ distance between two consecutive particles in the beginning of BLMS

$I_{v_{x}}[$ ] Turbulence Intensity

$l[\mathrm{~m}]$ NETmix channel length

$n i$ [ ] number of iterations of each BLMS injection

$n p$ [ ] number of particles

$n_{b}^{l}$ [ ] number of blue particles that exit the mixing chamber on the left side

$n_{b}^{r}$ [ ] number of blue particles that exit the mixing chamber on the right side

$n_{r}^{l}$ [ ] number of red particles that exit the mixing chamber on the left side

$n_{r}^{r}$ [ ] number of red particles that exit the mixing chamber on the right side

$n t$ [ ] total number of BLMS injections

$P_{b}^{l}$ [ ] exit conditional probability of blue particles exiting the mixing chamber on the left side

$P_{b}^{r}$ [ ] exit conditional probability of blue particles exiting the mixing chamber on the right side
$P_{r}^{l}$ [ ] exit conditional probability of red particles exiting the mixing chamber on the left side

$P_{r}^{r}$ [ ] exit conditional probability of red particles exiting the mixing chamber on the right side

$t_{0}[\mathrm{~s}]$ initial flowtime of BLMS

$x[\mathrm{~m}] x$-coordinate

$\mathbf{x 0}[\mathrm{m}]$ vector with initial $x$-coordinates in BLMS

$\mathbf{X}[\mathrm{m}]$ vector of the positions of the particles

$y[\mathrm{~m}] y$-coordinate.

\section{Greek letters}

$\Delta A\left[\mathrm{~m}^{2}\right]$ difference between consecutive areas $A_{i}$

$\Delta t[\mathrm{~s}]$ time-step size

$\Delta x[\mathrm{~m}]$ maximum hedge of NETmix mesh elements

$\tau[\mathrm{s}]$ residence time

$\rho\left[\mathrm{kg} \mathrm{m}^{-3}\right]$ fluid's density

$\mu$ [mPa.s] fluid's viscosity

$\omega[\mathrm{m}]$ NETmix geometry depth

$v\left[\mathrm{~m} \mathrm{~s}^{-1}\right]$ velocity

$v_{x}\left[\mathrm{~m} \mathrm{~s}^{-1}\right] x$-velocity

$v_{y}\left[\mathrm{~m} \mathrm{~s}^{-1}\right] y$-velocity

$\boldsymbol{v}\left[\mathrm{m} \mathrm{s}^{-1}\right]$ matrix with velocity vectors

$v 0\left[\mathrm{~m} \mathrm{~s}^{-1}\right]$ vector with initial $x$-velocities in BLMS

$v_{i n j}\left[\mathrm{~m} \mathrm{~s}^{-1}\right]$ velocity of injection. 